\title{
Association Mapping of Rice Germplasm Accessions for qDTY 1.1 and qDTY 12.1
}

\author{
Parminder Singh Saini ${ }^{1 *}$, Ritu R. Saxena ${ }^{1}$, Suman Rawte ${ }^{1}$, Sunil K. Verma ${ }^{1}$, \\ Ravi R. Saxena ${ }^{3}$ and S. B. Verulkar ${ }^{2}$ \\ ${ }^{1}$ Department of Genetics and Plant Breeding, \\ ${ }^{2}$ Department of Plant Molecular Biology and Biotechnology, \\ ${ }^{3}$ Department of Agricultural Statistics and Social Science, IGKV, Raipur (C.G), India \\ *Corresponding author
}

Keywords

Drought, Candidate gene, Rice,

Association

mapping, GLM

model

Article Info

Accepted:

10 January 2021

Available Online:

10 February 2021

\section{A B S T R A C T}

Chhattisgarh state is renowned by the name Rice Bowl of India because rice is cultivated in maximal land area. The research study was carried out by using fifty-eight rice landraces at Research cum Instructional farm of IGKV, Raipur during Kharif 2019 under irrigated and rainfed condition in Randomized Block Design (RBD), with two replications. Using the GLM model, for grain yield RM 240 (C\#2), RM 3825 (C\#1), RM 5753 (C\#6) and RM 11 (C\#7) exhibited tight association in irrigated condition and in RM 511 (C\#12), RM 307 (C\#4) and RM 495 (C\#1) had tight linkage with grain yield in rainfed condition. Based on two markers i.e., RM 3825 and RM 511 twenty genotypes had both the QTL's that is qDTY1.1 and qDTY 12.1.

\section{Introduction}

Rice is a primary staple food in major countries and used widely as a nutritional source. As with increase in demand due to population increment lead to increase in its production, but mainly rainfed condition is present in India, due to which drought leads to loss in yield. The process can be made more efficient and rapid through markerassisted breeding, a well-known fast-track approach in crop improvement. QTLs have been identified for grain yield under drought with large effects against droughtsusceptible varieties. "A QTL, qDTY12.1, significantly associated with grain yield under reproductive of stage drought stress was identified on chromosome 12. qDTY12.1 is the only QTL reported so far in rice to have shown a large effect against multiple recipient genetic backgrounds as well as under highly diverse upland and lowland rice ecosystems. 
qDTY12.1 can be successfully introgressed to improve grain yield under drought of popular high-yielding but drought-susceptible lowland as well as upland adapted varieties following marker-assisted breeding.

Drought is one of the most important abiotic stresses leading threat to agricultural food production especially in rice cultivation hampering rice productivity in rainfed areas. In Asia, more than 23 million ha of ricegrowing area are rainfed (Pandey et al., 2007). Eastern India and adjoining areas occupy a large drought-affected area with an estimate of around 17 million ha (Huke and Huke 1997). The green revolution had little impact in rainfed ecosystems (Evenson et al., 2003). Farmers in these areas are growing popular varieties originally bred for irrigated ecosystems (Vikaram et al., 2011). The slow progress in developing rice varieties for drought-prone areas is mainly due to the complex nature of drought-tolerance mechanisms; large genotype $\times$ environment, QTL $\times$ environment and QTL $\times$ recipient genetic background interactions; and the absence of QTLs with a large and consistent effect against high-yielding but droughtsusceptible varieties. This becomes even more relevant when we see that qDTY12.1 as well as qDTY2.3 is contributed by IR7437146-1-1. Recently, candidate gene analysis has been carried out in this QTL region and several genes have been reported as putative candidate genes (Kohli et al., 2010 and Biswal et al., 2012). Swamy et al., 2011 carried out meta-QTL analysis and reported several candidate genes in the same region. qDTY12.1 could be efficiently used in marker-assisted breeding for the improvement of both lowland and upland rice varieties for drought stress." Candidate gene studies have been at the forefront of genetic association studies i.e. identifying risk variants associated with a particular disease. Candidate gene studies are most often selected as this approach is relatively cheap and quick to perform, and are focused on the selection of genes that have been in some way related to the disease previously and thus come with prior knowledge about gene function. The candidate gene approach begins with selection of a putative candidate gene based on its relevance in the mechanism of the disease (trait) being investigated (Kwon and Goate, 2000 and Patnala et al., 2013).

\section{Materials and Methods}

\section{Experimental site}

The present research work was regulate at Research cum Instructional farm Department of Genetics and Plant Breeding, College of agriculture, Indira Gandhi Agricultural University, Raipur, Chhattisgarh, during the Kharif season of 2019.

\section{Climate and weather}

Chhattisgarh is situated between $17^{\circ} 14^{\prime} \mathrm{N}$ and $24^{\circ} 45^{\prime} \mathrm{N}$ latitudes and $79^{\circ} 16^{\prime} \mathrm{E}$ and $84^{\circ} 15^{\prime}$ E longitudes. Raipur (C.G.) is lies at $21^{\circ} 16^{\prime} \mathrm{N}$ latitude and $81^{\circ} 36^{\prime} \mathrm{E}$ longitude with an altitude of $(289.60 \mathrm{~m})$. Overhead mean sea level. The maximum mean temperature was $32.30^{\circ} \mathrm{C} \&$ minimum mean temperature was $23.99^{\circ} \mathrm{C}$ during the crop development time. The overall total rainfall during crop growing period was $(1199.2 \mathrm{~mm})$. The highest rainfall received during August month was $(316.4 \mathrm{~mm})$.

\section{Materials and Methods}

Fifty-eight genotypes of rice were taken for this research (Table 1). The material was grown under irrigated (transplanted) and rainfed (direct seeded) condition in the wet season of 2019. Nurseries were raised and 21 days old seedlings were eventually transplanted in the field, in RBD with 2 
replications. R X R and P X P distance of 25 $\mathrm{cm} \mathrm{X} 15 \mathrm{~cm}$ and net plot area was $3 \mathrm{~m} \mathrm{~m} \mathrm{X}$ $1.5 \mathrm{~m}$. The crop was preserve under irrigated condition. Fertilizer dose @ of 50:40:30 kg per hectare (NPK) was applied.

Before transplanting the whole dose of potassium and phosphorus are applied and half dose of nitrogen are applied as a basal dose. The remaining dose of $\mathrm{N}$ was applied in 2 splits, 1st at the period of starting of tillering and 2nd, 1week after it. All the treatment was similar for agronomical practices.

\section{Observations recorded}

The observation was recorded on days to 50 $\%$ flowering, plant height $(\mathrm{cm})$, flag Leaf length $(\mathrm{cm})$, flag Leaf width $(\mathrm{cm})$, number of effective tiller per plant, panicle length $(\mathrm{cm}), 1000$ grain weight $(\mathrm{gm})$, number of filled grains (NFG), number of unfilled grain (NUFG), total number of grains, biological yield per plant (BYP) (g), harvesting index (HI) $(\%)$ and grain yield per plant (GYP) (g) in irrigated and rainfed condition as well.

\section{Statistical analysis}

The data was notable in favour to divergent phenotypic and quantitative traits on the 13 yield and panicle related character of rice accessions were subjected to the statistical analysis.

In this calculation mean values were succeed for all character for everyone genotype. These mean data are used to estimate the variability parameters. ANOVA is deliberated by using OPSTAT software.

\section{Molecular study}

For molecular characterization, fifty-eight rice germplasm accessions are used. For estimating the genetic diversity of rice germplasm molecular study was execute, which included "DNA isolation, quantification, dilution of DNA, PCR amplification using microsatellite primers, electrophoresis using polyacrylamide gel, scoring and analysis of data.

\section{Results and Discussion}

Total genomic DNA was extracted from 58 lines of rice using CTAB method (Zheng et al., 1995). Fresh and healthy leaves were used for extraction of DNA. The DNA samples were quantified by using Nano Drop Spectroscopy (NANODROP 2000c). The quantity of the samples was found in the range from 500-2000 $\eta \mathrm{g} / \mu \mathrm{l}$. DNA samples were then diluted with sterilized water such that the final concentration of DNA became 50 $\eta \mathrm{g} / \mu \mathrm{l}$ (Table 1-5).

\section{Development of genotypic data based on SSR Markers}

Genetic associations among 58 accessions were analyzed, based on phenotypic variation of yield traits with the help of 42 SSR markers covering all the chromosomes. A total of 74 alleles were amplified and the number of alleles per locus generated by each marker ranged from 1 to 4 alleles with an average number of 1.76 alleles per locus. Maximum number of alleles (4) was amplified by marker RM 413, RM 518 and RM 240 marker (Table 5).

\section{Polymorphism Information Content of SSR markers}

The PIC value across markers ranged from 0.00 to 0.73 with an average of 0.25 . Maximum PIC value was observed on chromosome $4(\mathrm{RM} 518=0.73)$ followed by RM 489 of chromosome 3 (0.70) Table 5 (Fig. 1). 
Table.1 List of germplasm accessions

\begin{tabular}{|c|c|c|c|}
\hline $\begin{array}{c}\text { Entry } \\
\text { numbe } \\
\mathbf{r}\end{array}$ & Name of entry & $\begin{array}{c}\text { Entry } \\
\text { numbe } \\
\mathbf{r}\end{array}$ & Name of entry \\
\hline $\mathbf{1}$ & & 30 & Manaw Thukha \\
\hline $\mathbf{2}$ & IRRI 154 & 31 & BR28 \\
\hline $\mathbf{3}$ & MINGHUI 63 & 32 & TN1 \\
\hline $\mathbf{4}$ & ZHENSHAN 97 B & 33 & IR6 \\
\hline $\mathbf{5}$ & IR 64-21 & 34 & GSR IR2-9-R1-SU3-Y2 \\
\hline $\mathbf{6}$ & IRBB 66 & 35 & Zanton::IRGC 31248-1 \\
\hline $\mathbf{7}$ & IR 78222-20-7-148-2-B-B-B-B & 36 & URAIBOOL::IRGC 52785- \\
\hline $\mathbf{8}$ & IR 69726-116-1-1 & 37 & Hokkai 188 \\
\hline $\mathbf{9}$ & IRRI147 & 38 & IR 126182-1-1-1 \\
\hline $\mathbf{1 0}$ & SANHUNGZHAN NO 2 & 39 & IR10F360 \\
\hline $\mathbf{1 1}$ & IR77186-122-2-2-3 & 40 & Sahel 108 \\
\hline $\mathbf{1 2}$ & IR77298-14-1-2-10 & 41 & Sahel 134 \\
\hline $\mathbf{1 3}$ & Sambha Mahsuri + Sub1 & 42 & Sahel 177 \\
\hline $\mathbf{1 4}$ & Supa & 43 & Giza 178 \\
\hline $\mathbf{1 5}$ & IRRI 104 & 44 & Moroberekan \\
\hline $\mathbf{1 6}$ & N 22::IRGC 19379-1 & 45 & DJ123 \\
\hline $\mathbf{1 7}$ & MTU1010 & 46 & Oryzica 1 \\
\hline $\mathbf{1 8}$ & Swarna & 47 & F50 \\
\hline $\mathbf{1 9}$ & Nanhi & 48 & TEQING \\
\hline $\mathbf{2 0}$ & Jasmine 85 & 49 & MG 2::IRGC 79837-1 \\
\hline $\mathbf{2 1}$ & Kinandang Patong & 50 & UPL RI 7::IRTP 9897-C1 \\
\hline $\mathbf{2 2}$ & Sadri & 51 & CT11891-2-2-7-M \\
\hline $\mathbf{2 3}$ & OM4900 & 52 & Oryzica sabana 10 \\
\hline $\mathbf{2 4}$ & IR 93340:14-B-21-17-12-1RGA-2RGA-1-B-B & 53 & Oryzica sabana 6 \\
\hline $\mathbf{2 5}$ & IR 93354:34-B-5-1-23-1RGA-2RGA-1-B-B & 54 & Oryzica Llanos 5 \\
\hline $\mathbf{2 6}$ & Khao Hlan On & 55 & Chhomrong Dhan \\
\hline $\mathbf{2 7}$ & IR13F167 & 56 & NSIC Rc240 \\
\hline $\mathbf{2 8}$ & IR84984-83-15-481-B & 57 & Jamir \\
\hline $\mathbf{2 9}$ & M202 & $\mathbf{5 8}$ & IR10M300 \\
\hline
\end{tabular}


Table.2 Significant marker-trait associations based on GLM model $(\mathrm{P}>0.05)$ in irrigated and rainfed condition during Kharif 2019

\begin{tabular}{|c|c|c|c|c|c|c|c|c|c|}
\hline Trait & Locus & C \# & $p_{-}$ & Rsq_ & Trait & Locus & C \# & $\mathbf{p}_{-}$ & Rsq \\
\hline & & & Marker & Marker & & & & Marker & Marke \\
\hline Irrigate & & & & & Rainfed & & & & \\
\hline \multirow[t]{3}{*}{ DTF } & RM 3825 & 1 & 0.0025 & 0.1536 & DTF & RM 3825 & 1 & $7.32 \mathrm{E}-05$ & 0.2478 \\
\hline & RM 11 & 7 & 0.0194 & 0.1379 & & RM 11 & 7 & $5.21 \mathrm{E}-04$ & 0.2454 \\
\hline & RM 5753 & 6 & 0.0246 & 0.1279 & & RM 518 & 4 & 0.0519 & 0.1647 \\
\hline \multirow[t]{3}{*}{ PH } & RM 6504 & 1 & 0.0168 & 0.1305 & $\mathrm{PH}$ & RM 240 & 2 & 0.0065 & 0.1802 \\
\hline & RM 28130 & 12 & 0.0233 & 0.0899 & & RM 413 & 4 & 0.0142 & 0.1844 \\
\hline & RM 240 & 2 & 0.0353 & 0.1249 & ET & RM 518 & 4 & 0.0252 & 0.1834 \\
\hline FLL & RM 28160 & 12 & 0.0235 & 0.0975 & FLL & RM 240 & 2 & 0.0164 & 0.1474 \\
\hline \multirow[t]{3}{*}{ FLW } & OSR 13 & 1 & 0.0016 & 0.2357 & & OSR 13 & 1 & 0.0404 & 0.1225 \\
\hline & RM 6504 & 1 & 0.0141 & 0.1372 & & RM 28160 & 12 & 0.0431 & 0.0836 \\
\hline & RM 3825 & 1 & 0.045 & 0.0703 & & RM 1261 & 12 & 0.0479 & 0.0872 \\
\hline \multirow[t]{4}{*}{ PL } & RM 3825 & 1 & 0.0085 & 0.1191 & FLW & RM 1261 & 12 & 0.0598 & 0.0818 \\
\hline & OSR 13 & 1 & 0.0166 & 0.1594 & PL & OSR 13 & 1 & 0.0164 & 0.1588 \\
\hline & RM 489 & 3 & 0.0424 & 0.1303 & & RM 240 & 2 & 0.0213 & 0.1393 \\
\hline & RM 5753 & 6 & 0.0462 & 0.1075 & & RM 489 & 3 & 0.0246 & 0.1538 \\
\hline TGW & RM 236 & 4 & 0.0598 & 0.0731 & TGW & RM 1261 & 12 & 0.0078 & 0.118 \\
\hline \multirow[t]{4}{*}{ GY } & RM 240 & 2 & 0.0075 & 0.1779 & & RM 5753 & 6 & 0.0385 & 0.0923 \\
\hline & RM 3825 & 1 & 0.0177 & 0.0978 & GY & RM 511 & 12 & 0.0397 & 0.0889 \\
\hline & RM 5753 & 6 & 0.0245 & 0.128 & & RM 307 & 4 & 0.0434 & 0.0714 \\
\hline & RM 11 & 7 & 0.0499 & 0.1067 & & RM 495 & 1 & 0.0482 & 0.0726 \\
\hline \multirow[t]{2}{*}{ BY } & RM 5753 & 6 & 0.0366 & 0.1152 & BY & RM 495 & 1 & 0.065 & 0.0495 \\
\hline & RM 3825 & 1 & 0.0529 & 0.0664 & HY & RM 1261 & 12 & 0.0138 & 0.1279 \\
\hline HY & RM 240 & 2 & 0.0263 & 0.1354 & & RM 236 & 4 & 0.0357 & 0.0848 \\
\hline NFG & RM 11 & 7 & 0.0263 & 0.1283 & NFG & RM 125 & 7 & 0.0134 & 0.1543 \\
\hline \multirow[t]{2}{*}{ NUFG } & RM 240 & 2 & 0.0609 & 0.0977 & & RM 489 & 3 & 0.0571 & 0.1179 \\
\hline & RM 28130 & 12 & 0.0884 & 0.0455 & NUFG & OSR 13 & 1 & 0.0015 & 0.2404 \\
\hline \multirow[t]{6}{*}{ TNG } & RM 11 & 7 & 0.0232 & 0.1319 & & RM 125 & 7 & 0.0277 & 0.1312 \\
\hline & & & & & TNG & RM 125 & 7 & 0.0044 & 0.191 \\
\hline & & & & & & RM 11 & 7 & 0.0273 & 0.1261 \\
\hline & & & & & & RM 489 & 3 & 0.052 & 0.1212 \\
\hline & & & & & & OSR 13 & 1 & 0.0659 & 0.1089 \\
\hline & & & & & & RM 3520 & 1 & 0.071 & 0.0609 \\
\hline
\end{tabular}

DTF = Days to flowering; PH = plant height $(\mathrm{cm}) ; \mathrm{NT}=$ Number of tillers; FLL = Flag leaf Length $(\mathrm{cm}) ;$ FLW $=$ Flag leaf width $(\mathrm{cm}) ; \mathrm{PL}=$ Panicle Length $(\mathrm{cm}) ; \mathrm{TGW}=$ Thousand grain weight $(\mathrm{g}) ; \mathrm{NFG}=$ Number of filled grains; NUFG $=$ Number of unfilled grains; $\mathrm{TNG}=$ Total Number of grains; BYP = Biological yield per plant $(\mathrm{g})$; $\mathrm{HI}=$ Harvest index $(\%)$ GYP $=$ Grain yield per plant $(\mathrm{g})$ 
Table.3 Significant marker-trait associations based on MLM model $(\mathrm{P}>0.05)$ in irrigated and rainfed condition during Kharif 2019

\begin{tabular}{|c|c|c|c|c|c|c|c|c|c|}
\hline Trait & Locus & C \# & p_Marke & Rsq_ & Trait & Locus & C \# & p_Marke & Rsq $_{-}$ \\
\hline & & & & Marker & & & & & Marke \\
\hline Irrigate & & & & & Rainfed & & & & \\
\hline \multirow[t]{3}{*}{ DTF } & RM 3825 & 1 & 0.0025 & 0.1536 & DTF & RM 3825 & 1 & 7.32E-05 & 0.2478 \\
\hline & RM 11 & 7 & 0.0194 & 0.1379 & & RM 11 & 1 & $5.21 \mathrm{E}-04$ & 0.2454 \\
\hline & RM 5753 & 6 & 0.0246 & 0.1279 & $\mathrm{PH}$ & RM 413 & 4 & 0.0109 & 0.1844 \\
\hline \multirow[t]{3}{*}{ PH } & RM 6504 & 1 & 0.0165 & 0.1305 & & RM 240 & 2 & 0.0231 & 0.1802 \\
\hline & RM 28130 & 12 & 0.0233 & 0.0899 & NT & RM 518 & 4 & 0.0385 & 0.1834 \\
\hline & RM 240 & 2 & 0.0353 & 0.1249 & FLL & RM 240 & 2 & 0.0164 & 0.1474 \\
\hline NT & RM 6504 & 1 & 0.0604 & 0.0825 & & OSR 13 & 1 & 0.0404 & 0.1225 \\
\hline \multirow[t]{2}{*}{ FLL } & RM 28160 & 12 & 0.0235 & 0.0975 & FLW & RM 1261 & 12 & 0.0598 & 0.0818 \\
\hline & OSR 13 & 1 & 0.068 & 0.1019 & PL & OSR 13 & 1 & 0.0366 & 0.1588 \\
\hline \multirow[t]{3}{*}{ FLW } & OSR 13 & 1 & 0.0016 & 0.2357 & & RM 240 & 2 & 0.039 & 0.1393 \\
\hline & RM 6504 & 1 & 0.0141 & 0.1372 & TGW & RM 1261 & 12 & 0.0257 & 0.118 \\
\hline & RM 3825 & 1 & 0.045 & 0.0703 & NFG & RM 125 & 7 & 0.0145 & 0.1543 \\
\hline \multirow[t]{3}{*}{ PL } & RM 3825 & 1 & 0.0085 & 0.1191 & NUFG & OSR 13 & 1 & 0.0015 & 0.2404 \\
\hline & OSR 13 & 1 & 0.0166 & 0.1594 & & RM 125 & 7 & 0.0277 & 0.1312 \\
\hline & RM 5753 & 6 & 0.056 & 0.1075 & TNG & RM 125 & 7 & 0.0049 & 0.191 \\
\hline TGW & RM 236 & 2 & 0.0598 & 0.0731 & & RM 11 & 7 & 0.0274 & 0.1261 \\
\hline GY & RM 240 & 2 & 0.0075 & 0.1779 & & OSR 13 & 1 & 0.0539 & 0.1089 \\
\hline BY & RM 5753 & 6 & 0.0522 & 0.1152 & & & & & \\
\hline NFG & RM 11 & 7 & 0.0263 & 0.1283 & & & & & \\
\hline TNG & RM 11 & 7 & 0.0232 & 0.1319 & & & & & \\
\hline
\end{tabular}

DTF = Days to flowering; PH = plant height $(\mathrm{cm}) ; \mathrm{NT}=$ Number of tillers; FLL = Flag leaf Length $(\mathrm{cm}) ;$ FLW $=$ Flag leaf width $(\mathrm{cm}) ; \mathrm{PL}=$ Panicle Length $(\mathrm{cm}) ; \mathrm{TGW}=$ Thousand grain weight $(\mathrm{g}) ; \mathrm{NFG}=$ Number of filled grains; NUFG $=$ Number of unfilled grains; $\mathrm{TNG}=$ Total Number of grains; $\mathrm{BYP}=$ Biological yield per plant $(\mathrm{g})$; $\mathrm{HI}=$ Harvest index $(\%) ; \mathrm{GYP}=$ Grain yield per plant $(\mathrm{g})$ 
Table.4 List of genotypes having qDTY 1.1 and qDTY 12.1 or both based on markers position

\begin{tabular}{|c|c|c|c|c|c|}
\hline S. No & $\begin{array}{l}\text { Markers } \\
\text { for } \\
\text { qDTY } 1.1\end{array}$ & $\begin{array}{l}\text { Band size } \\
\quad \text { (bp) }\end{array}$ & $\begin{array}{c}\text { PIC } \\
\text { VALUE }\end{array}$ & $\begin{array}{l}\text { Number of } \\
\text { genotypes }\end{array}$ & Name of genotypes \\
\hline \multirow[t]{2}{*}{1} & RM 3825 & 147 & 0.26 & 32 & $\begin{array}{l}\text { IRRI 154, MINGHUI 63, ZHENSHAN } 97 \text { B, IR 64-21, IRBB 66, IRRI147, SANHUANGZHA } \\
\text { NO 2, IR77186-122-2-2-3, IR77298-14-1-2-10, Supa, IRRI 104, N 22::IRGC 19379-1, } \\
\text { MTU1010, Jasmine 85, Sadri, OM4900, IR 95042:13-B-7-11-15-3, IR 93340:14-B-21-17-12- } \\
\text { 1RGA-2RGA-1-B-B, DJ123, Oryzica 1, F50, TEQING, MG 2::IRGC } \\
\text { 79837-1, UPL RI 7::IRTP 9897-C1 CT11891-2-2-7-M, Oryzicasabana 10, Oryzicasabana 6, } \\
\text { Oryzica Llanos 5, ChhomrongDhan, NSIC Rc240, Jamir, IR10M300 }\end{array}$ \\
\hline & $\begin{array}{l}\text { Markers } \\
\text { for } \\
\text { qDTY } 12.1\end{array}$ & $\begin{array}{l}\text { Band size } \\
\quad(\text { bp) }\end{array}$ & $\begin{array}{c}\text { PIC } \\
\text { VALUE }\end{array}$ & & \\
\hline 2 & RM 511 & 130 & 0.44 & 43 & $\begin{array}{l}\text { MINGHUI 63, ZHENSHAN } 97 \text { B, IR 64-21, IRBB 66, IR 69726-116-1-1, SANHUANGZHA } \\
\text { NO 2, SambhaMahsuri + Sub1, N 22::IRGC 19379-1, MTU1010, Nanhi, Jasmine 85, } \\
\text { KinandangPatong, Sadri, OM4900, IR 95042:13-B-7-11-15-3, IR } \\
\text { 93340:14-B-21-17-12-1RGA-2RGA-1-B-B, IR 93354:34-B-5-1-23-1RGA-2RGA-1-B-B, KhaoHlan } \\
\text { On, IR13F167, IR84984-83-15-481-B, M202, ManawThukha, BR28, TN1, GSR IR2-9-R1-SU3- } \\
\text { Y2, Zanton::IRGC 31248-1, URAIBOOL::IRGC 52785-1, Hokkai 188, IR } \\
\text { 126182-1-1-1, IR10F360, Sahel 108, Sahel 134, Sahel 177, Giza 178, Moroberekan, TEQING, } \\
\text { CT11891-2-2-7-M, Oryzicasabana 10, Oryzica Llanos 5, ChhomrongDhan, NSIC Rc240, Jamir, } \\
\text { IR10M300 }\end{array}$ \\
\hline \multicolumn{5}{|c|}{$\begin{array}{l}\text { Name of common genotype for both QTL (1.1 and 12.1) } \\
\text { based on two markers namely, RM } 3825 \text { and RM } 511\end{array}$} & $\begin{array}{c}\text { Minghui } 63 \text {, Zhenshan } 97 \text { b, IR 64-21, IRBB 66, Sanhuangzha No 2, N22::IRGC 19379-1, } \\
\text { MTU 1010, Jasmine 85, Sadri, OM 4900, IR95042:13-B-7-11-15-3, } \\
\text { IR93340:14-B-21-17-12-1RGA-2RGA-1-B-B, Teqing, CT11891-2-2-7-M, Oryzica sabana 10, } \\
\text { Oryzica Llanos 5, Chhomrong Dhan, NSCIC Rc240, Jamir, IR10M300 }\end{array}$ \\
\hline
\end{tabular}


Table.5 List of 42 microsatellite markers with their chromosome locations, number of alleles, allele size and PIC value found among 58 rice accessions.

\begin{tabular}{|c|c|c|c|c|c|c|c|}
\hline MARKERS & Forward motif & Reverse motif & C\# & $\begin{array}{l}\text { Position } \\
\text { (cM) }\end{array}$ & $\begin{array}{l}\text { Amplicon } \\
\text { Size (bp) }\end{array}$ & $\begin{array}{c}\text { PIC } \\
\text { value }\end{array}$ & $\begin{array}{l}\text { No. of } \\
\text { Alleles }\end{array}$ \\
\hline RM 413 & GGCGATTCTTGGATGAAGAG & TCCCCACCAATCTTGTCTTC & 5 & 26.7 & $71-114$ & 0.48 & 4 \\
\hline OSR 13 & CATTTGTGCGTCACGGAGTA & AGCCACAGCGCCCATCTCTC & 3 & 53.1 & $85-122$ & 0.51 & 1 \\
\hline RM 133 & TTGGATTGTTTTGCTGGCTCGC & GGAACACGGGGTCGGAAGCGAC & 6 & 0 & 230 & 0.00 & 1 \\
\hline RM 489 & ACTTGAGACGATCGGACACC & TCACCCATGGATGTTGTCAG & 3 & 29.2 & 271 & 0.70 & 3 \\
\hline RM 161 & TGCAGATGAGAAGCGGCGCCTC & TGTGTCATCAGACGGCGCTCCG & 5 & 96.9 & $154-187$ & 0.00 & 1 \\
\hline RM 125 & ATCAGCAGCCATGGCAGCGACC & AGGGGATCATGTGCCGAAGGCC & 7 & 24.8 & $105-147$ & 0.45 & 3 \\
\hline RM 11 & TCTCСТCTTCCCCCGATC & ATAGCGGGCGAGGCTTAG & 7 & 47 & $118-151$ & 0.46 & 3 \\
\hline RM 307 & GTACTACCGACCTACCGTTCAC & CTGCTATGCATGAACTGCTC & 4 & 0 & $116-191$ & 0.51 & 2 \\
\hline RM 287 & TTCCCTGTTAAGAGAGAAATC & GTGTATTTGGTGAAAGCAAC & 11 & 68.6 & 118 & 0.68 & 2 \\
\hline RM 316 & CTAGTTGGGCATACGATGGC & ACGCTTATATGTTACGTCAAC & 9 & 1.8 & $194-216$ & 0.00 & 1 \\
\hline RM 536 & TCTCTCCTCTTGTTTGGCTC & ACACACCAACACGACCACAC & 11 & 55.1 & 243 & 0.00 & 1 \\
\hline RM 520 & AGGAGCAAGAAAAGTTCCCC & GCCAATGTGTGACGCAATAG & 3 & 191.6 & $50-247$ & 0.16 & 2 \\
\hline RM 236 & GCGCTGGTGGAAAATGAG & GGCATCCCTCTTTGATTCCTC & 2 & 175 & $105-191$ & 0.44 & 2 \\
\hline RM 511 & CTTCGATCCGGTGACGAC & AACGAAAGCGAAGCTGTCTC & 12 & 59.8 & $50-130$ & 0.44 & 2 \\
\hline RM 3739 & AGTTGCGCAGCTAATCGATC & AAGATCCAACGGGTTCTGTG & 12 & 119.2 & 110 & 0.00 & 1 \\
\hline RM 12010 & GGACCGAATCATGAGACAATGG & TTGATGACGTCAAGCGAGAAACC & 1 & 38.91 & $194-284$ & 0.00 & 1 \\
\hline RM 3825 & AAAGCCCCCAAAAGCAGTAC & GTGAAACTCTGGGGTGTTCG & 1 & 143.7 & $50-147$ & 0.26 & 2 \\
\hline RM 28160 & AACCACCATCCСАCТCCСAACC & CTTATGCCACCACTAACTCCCAAACC & 12 & 17.47 & $136-249$ & 0.56 & 2 \\
\hline RM 28199 & CGGCTTAGGGAGCGTCTGTAGG & GCATGCTAGTATGGCCACCATATTCC & 12 & 18.15 & 179 & 0.00 & 1 \\
\hline RM 452 & CTGATCGAGAGCGTTAAGGG & GGGATCAAACCACGTTTCTG & 2 & 58.4 & 209 & 0.00 & 1 \\
\hline RM 472 & CCATGGCCTGAGAGAGAGAG & AGCTAAATGGCCATACGGTG & 1 & 171.6 & 296 & 0.00 & 1 \\
\hline RM 416 & GGGAGTTAGGGTTTTGGAGC & TCCAGTTTCACACTGCTTCG & 3 & 191.6 & 114 & 0.00 & 1 \\
\hline RM 518 & СТСТTСАСТСАСТСАССАТGG & ATCCATCTGGAGCAAGCAAC & 4 & 25.5 & $135-171$ & 0.73 & 4 \\
\hline RM 240 & CCTTAATGGGTAGTGTGCAC & TGTAACCATTCCTTCCATCC & 2 & 158 & $50-132$ & 0.54 & 4 \\
\hline RM 6504 & GAGAGGCGGTAGTATGCTCG & TCTCCAATTTCACCGTCACC & 1 & 154.6 & $50-116$ & 0.59 & 3 \\
\hline RM 5753 & AACATGCTCAACTTCTGGGC & GCTAGGTACGATCCAGCTGC & 6 & 148 & 201 & 0.53 & 3 \\
\hline
\end{tabular}




\begin{tabular}{|c|c|c|c|c|c|c|c|}
\hline MARKERS & Forward motif & Reverse motif & C\# & $\begin{array}{c}\text { Position } \\
\text { (cM) }\end{array}$ & $\begin{array}{l}\text { Amplicon } \\
\text { Size (bp) }\end{array}$ & $\begin{array}{c}\text { PIC } \\
\text { value }\end{array}$ & $\begin{array}{l}\text { No. of } \\
\text { Alleles }\end{array}$ \\
\hline RM 1361 & ATTCTCTCCGCCTAAACAAC & TTCTCGTGCACAGTTAATACC & 1 & 155.2 & 214 & 0.00 & 1 \\
\hline RM 3520 & GAGGCTATATGCTCATGCTC & AAACCTGCAAATGCACAG & 1 & 159.6 & $150-178$ & 0.39 & 2 \\
\hline RM 28130 & CAGCAGACGTTCCGGTTCTACTCG & AGGACGGTGGTGGTGATCTGG & 12 & 79.7 & $60-175$ & 0.26 & 2 \\
\hline RM 28089 & GGGAGGACACCTGTGTAAGTAGG & GGTTCAAATGAGACCCAATTCC & 12 & 15.41 & $150-260$ & 0.19 & 2 \\
\hline RM 495 & AATCCAAGGTGCAGAGATGG & CAACGATGACGAACACAACC & 1 & 2.8 & 159 & 0.55 & 2 \\
\hline RM 486 & ССССССТСТСТСТСТСТСТС & TAGCCACATCAACAGCTTGC & 1 & 153.5 & 104 & 0.00 & 1 \\
\hline RM 28048 & TTCAGCCGATCCATTCAATTCC & GCTATTGGCCGGAAAGTAGTTAGC & 12 & 14.10 & 93 & 0.00 & 1 \\
\hline RM 28076 & GGGACTTGGGACCAGTTTATGG & TCAGGTCTGTTGGATTCCATGC & 12 & 15.13 & 289 & 0.00 & 1 \\
\hline RM 28086 & CCCGCTGCAGCAGTTTATTGAGG & GATCTGGTACCTGCATGGGTTGC & 12 & 15.33 & 387 & 0.00 & 1 \\
\hline RM 283 & GTCTACATGTACCCTTGTTGGG & CGGCATGAGAGTCTGTGATG & 1 & 31.4 & 151 & 0.00 & 1 \\
\hline RM 19 & CAAAAACAGAGCAGATGAC & CTCAAGATGGACGCCAAGA & 12 & 20.9 & 226 & 0.00 & 1 \\
\hline RM 431 & TCCTGCGAACTGAAGAGTTG & AGAGCAAAACCCTGGTTCAC & 1 & 178.3 & 251 & 0.00 & 1 \\
\hline RM 474 & AAGATGTACGGGTGGCATTC & TATGAGCTGGTGAGCAATGG & 10 & 0 & 252 & 0.00 & 1 \\
\hline
\end{tabular}


Fig.1 Graphical representation of PIC value of 22 polymorphic SSR markers

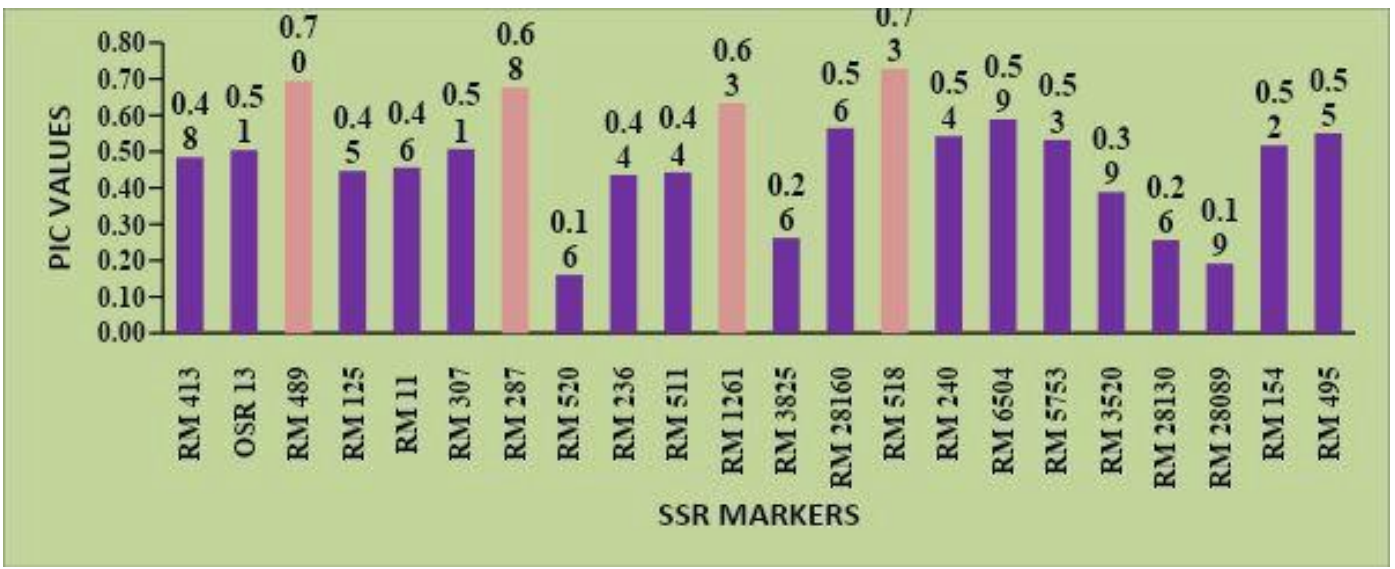

Fig.2 Estimation of $K$ and population structure. Changes in $\Delta K$ value with the number of subpopulations

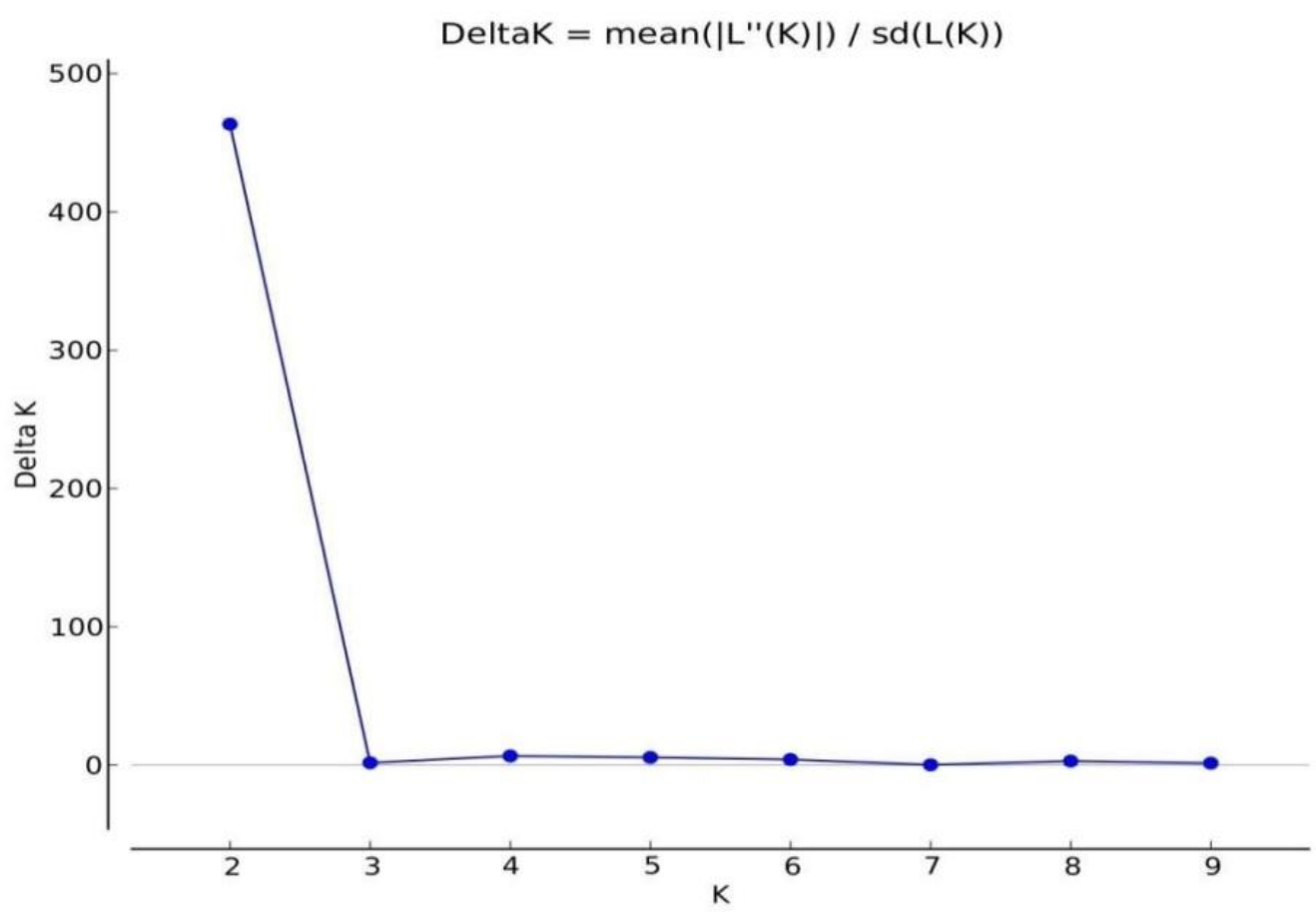

Optimum number of $K=2$ 
Fig.3 Bar plot showing the population structure of 58 rice germplasm accessions based on SSR markers at $\mathrm{K}=2$
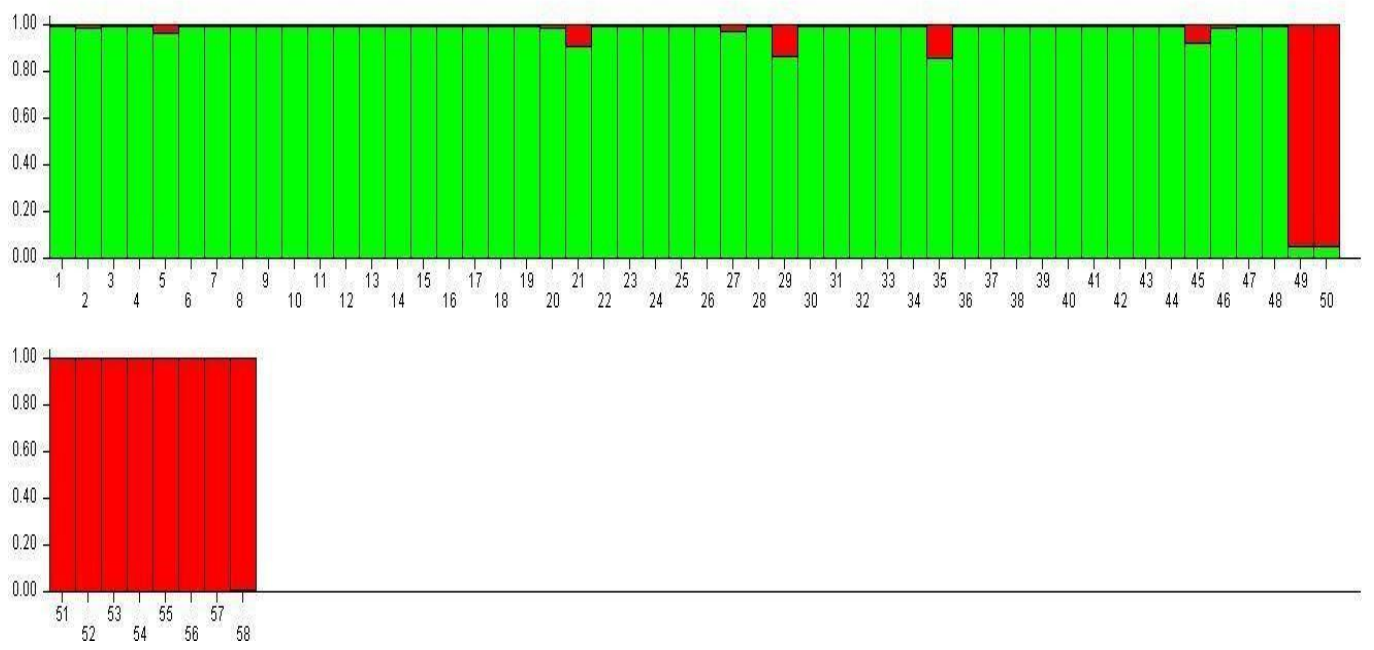

Structure and TASSEL analysis Population Structure analysis

The Bayesion model-based STRUCTURE v2.3.4 program was used to infer population structure of rice genotypes. The 58 lines were divided into two sub groups (Fig. 2) based on the result of Structure Harvester, as delta $\mathrm{K}$ kinship was highest at $\mathrm{K}=2$. With population inferred ancestry $(\mathrm{Q})=0.80$, forty three lines were assigned to subgroup POP1, rest 8 lines were assigned to subgroup POP2 and seven genotypes/ lines namely, IRBB 66 (5), Sadri (21), M 202 (29), Zanton::IRGC 31248-1 (35), DJ123 (45), MG2::IRGC 79837-1(49) and UPLRI7:: IRTP9897-C1 (50) were assigned to admixture (AD) which has less than $<0.80$ inferred ancestry (Fig. 3).

Courtois et al., (2012) has successfully detected two subgroups in their study population and assigned rice varieties into two groups with few admixture lines. Our results are also in conformity with the findings of Borba (2010) suggesting that using structure analysis, the accessions were sub divided into two panels. Likewise, the association of yield traits with SSR markers was undertaken with GLM model, with markers and sub population as fixed factors, and kinship matrix as random factor.

\section{Marker-trait association}

Association analysis between SSR markers and thirteen agronomic and yield attributing traits was carried out using GLM model over the 58 rice germplasm lines. The marker trait associations based on general linear model (GLM) in irrigated and rainfed conditions are presented in Table 2.

The results indicated that 26 and 31 markers were found to be associated with all the traits in irrigated and rainfed conditions respectively. For days to flowering, RM 3825 (C\# 1) and RM 11 (C\#7) were found to have tight association in irrigated and rainfed condition. However, RM 5753 (C\#6; irrigated) and RM 518 (C\#4; rainfed) also possessed tight linkage with days to flowering. Taking MLM model into consideration in irrigated condition, RM 240 $(\mathrm{C \# 2})$ showed tight linkage with grain yield, presented in Table 3. 


\section{References}

Borba, T.C., Brondani, R.V., Breseghello, F., Coelho, A.S.G., Mendonça, J.A., Rangel, P.H.N., Brondani, C. 2010. Association mapping for yield and grain quality traits in rice (Oryza sativa L.). Genet Mol Biol 33(3): 515-524.

Courtois, B., Audebert, A., Dardou, A., Roques, S. and Ghneim-Herrera., T. 2013. Genome wide association mapping of root traits in a japonica rice panel. PLoS One 8(11):78037805.

Evenson, R.E. and Gollin, D. 2003. Assessing the impact of the green revolution, 1960 to 2000. Science 300: 758-762.

Kohli, A., Narciso, J., Oane, R., Popluechai, S. and Kumar, A. 2010. Identification of major candidate genes in a large effect QTL for rice yield under drought stress, Paper presented at International Rice Congress, Hanoi, Vietnam, 9-11.

Kwon, J.M. and Goate, A.M. 2000. The candidate gene approach. Alcohol Res Health. 24(3): 164-168.

Pandey, S., Bhandari, H.S. and Hardy, B., 2007. Economic costs of drought and rice farmers' coping mechanisms: a cross-country comparative analysis. Int. Rice Res. Inst.

Patnala, R., Clements, J. and Batra, J. Candidate gene association studies: comprehensive guide to useful in silico tools. BMC Genet 14, 39 (2013).

Richharia, R.H. 1979. An aspect of genetic diversity in rice. Oryza. 16(1): 1-31.

Swamy, B.M., Vikram, P., Dixit, S., Ahmed, H.U. and Kumar, A., 2011. Metaanalysis of grain yield QTL identified during agricultural drought in grasses showed consensus. BMC genomics, 12(1), p.319.

Vikram, P., Swamy, B., Dixit, S., Ahmed, H., Cruz, M.T.S., Singh, A. and Kumar, A. 2011. qDTY1.1, a major QTL for rice grain yield under reproductive stage drought stress with a consistent effect in multiple elite genetic backgrounds. BMC Genet.12:89. 7289.

Zheng, K., Subudhi, P.K., Domingo, J., Magpantay, G. and Huang, N. 1995. Rapid DNA isolation for marker assisted selection in rice breeding. Rice Genet. Newsletter 12: 255-258.

\section{How to cite this article:}

Parminder Singh Saini, Ritu R. Saxena, Suman Rawte, Sunil K. Verma, Ravi R. Saxena and Verulkar, S. B. 2021. Association Mapping of Rice Germplasm Accessions for qDTY 1.1 and qDTY 12.1. Int.J.Curr.Microbiol.App.Sci. 10(02): 1418-1429.

doi: https://doi.org/10.20546/ijcmas.2021.1002.170 